\title{
Simultaneous Quality and Flow Rate Monitoring of Diesel Exhaust Fluid by Using A Platinum Thin Film Sensor
}

\author{
Ralf E. Bernhardsgrütter ${ }^{1,2}$, Christoph Hepp ${ }^{1}$, Katrin Schmitt²,3, Jürgen Wöllenstein ${ }^{2,3}$ \\ 1 Innovative Sensor Technology IST AG, Ebnat-Kappel, Switzerland, \\ ${ }^{2}$ Department of Microsystems Engineering IMTEK, University of Freiburg, Freiburg, Germany \\ ${ }^{3}$ Fraunhofer Institute for Physical Measurement Technique IPM, Freiburg, Germany \\ ralf.bernhardsgruetter@ist-ag.com
}

\begin{abstract}
Summary:
The monitoring of the quality and the flow rate of the diesel exhaust fluid is essential for an efficient selective catalytic reduction in diesel combustion. This article presents a platinum thin film sensor using a combination of the constant temperature anemometry and the $3 \omega$-method to measure continuously the urea content and the flow rate with compensated fluid dependence. The urea content can be determined within $3 \%$ by weight and the flow rate within $4 \%$ full scale. The presented method allows continuous monitoring of two main parameter in the selective catalytic reduction.
\end{abstract}

Keywords: Diesel exhaust fluid, flow measurement, quality monitoring, $3 \omega$-method, anemometry

\section{Motivation and Background}

The selective catalytic reduction (SCR), an aftertreatment method to reduce the emission in diesel combustion engines, can convert the harmful detrimental nitrous oxide into water and nitrogen by using a so-called diesel exhaust fluid (DEF), known as the trademark "AdBlue". The DEF consists of $32.5 \%$ urea and $67.5 \%$ water. The ratio of urea and water is crucial for an optimal aftertreatment and an inappropriate ratio breaks the SCR down and the emission reduction fails.

Reported DEF quality sensors, based on optical principles [1], ultrasonic [2] and sinusoidal heating measurement [3], are not sensitive to flow or are driven at non-flow condition due to a cross correlation between flow and fluid composition.

The claims of this paper are: A method using a combination of the constant temperature anemometry (CTA) and the $3 \omega$-method to (1) measure simultaneously flow and (2) determine the urea concentration. (3) The measurement deviations are better than $4 \%$ full scale for the flow measurement and better than $3 \%$ for the urea concentration measurement. (4) The sensor is suitable for on-the-fly measurements in e.g. vehicles.

\section{Method}

On one side, the CTA [4] is a well-known flow measurement method. However, the state-ofthe-art thermal flow measurement are highly dependent on the fluid and the sensor must be calibrated for each fluid. On the other side, the $3 \omega$ method is a well-known method to measure thermal conductivity and heat capacity [5], but the signal has usually a flow cross sensitivity. A crucial quantity in the field of the $3 \omega$-method is the penetration depth $\delta$ which depends on the surrounding, e.g. thermal conductivity and flow, and on the frequency $\omega$ as following,

$\delta \propto \sqrt{\frac{1}{\omega}}$.

Because the flow velocity changes perpendicular to the flow direction and is zero at the wall boundaries, a frequency range might exist where the fluid dependence is still visible, and dominant compared to the flow dependence. This article shows that such a frequency exists and can be used to measure the composition of a binary mixture and to compensate the fluid dependence of the CTA-measurement.

\section{Experiment \& Result}

The investigated sensor (see figure 1) bases on platinum thin film technology.

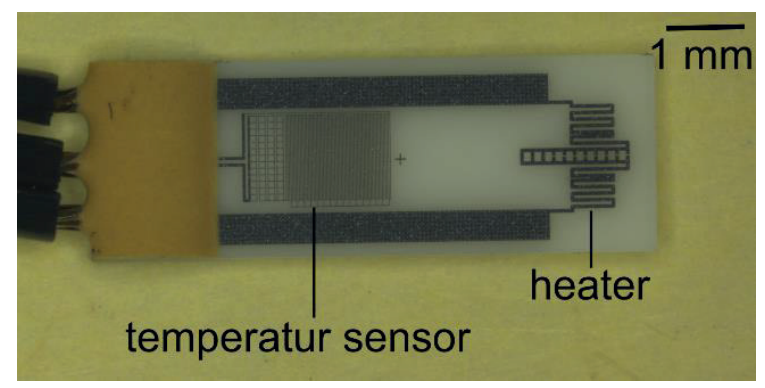

Figure 1: Platinum thin film element consists of a temperature sensor with $1200 \Omega$ at $0^{\circ} \mathrm{C}$ and a heating element with $45 \Omega$ at $0^{\circ} \mathrm{C}$. 
The sensor consists of two structure, a heater and a temperature sensor. Both structures have a temperature-to-resistance coefficient of 3900 $\mathrm{ppm} / \mathrm{K}$. The setup consists of an electronic circuit able to do the CTA-mode and the $3 \omega$-mode.

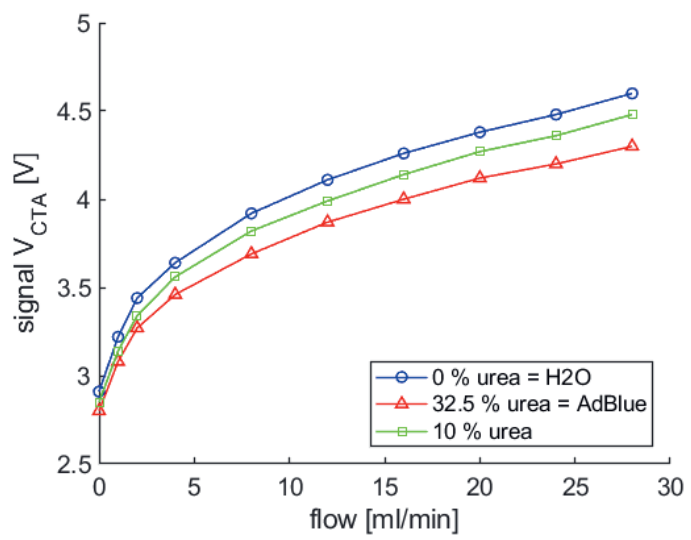

Figure 2: CTA-signal for different fluids, namely water, a urea-water mixture of $32.5 \%$ urea and a urea-water mixture of $10 \%$ urea as function of flow.

The experiment was done for three mixtures, namely water, a urea-water mixture of $32.5 \%$ urea (pure DEF) and a urea-water mixture of 10 $\%$ urea. The sensor calibration was done with pure DEF. Figure 2 shows the uncompensated CTA-signal for these fluids and the fluid dependence is highly visible which leads to a measurement error of the linearized flow of around $50 \%$ full scale in a state-of-the-art thermal flow meter.

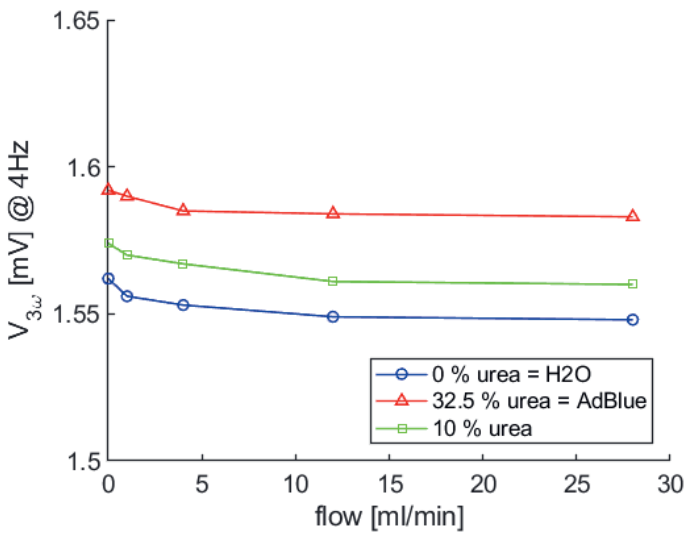

Figure 3: 3w-method signal for a drive frequency of 4 $\mathrm{Hz}$ as function of flow. The fluid dependence is dominant compared to the flow dependence.

The $3 \omega$-method investigations showed that the flow dependence is reduced for increasing drive frequency due to the decrease of the penetration depth (see eq. 1), however, the signal strength reduces as well. Therefore, a trade-off between flow dependence and signal strength exists. For the given setup and at a drive frequency of $4 \mathrm{~Hz}$, the $3 \omega$-signal (see figure 3 ) is still fluid dependent, but the flow dependence is strongly reduced. Given the base lines of water and pure
DEF, the urea content of the $10 \%$-mixture is determined to be between $10 \%$ and $13 \%$ urea for the entire flow range.

Using the extracted information of the $3 \omega$ method, the fluid dependence can be compensated. In this investigation, the dependence between the $3 \omega$-signal of a mixture at the given drive frequency of $4 \mathrm{~Hz}$ and its urea concentration is approximated to be linear. Figure 4 shows the compensated and linearized flow signal and the deviation with respect to the calibration with pure DEF. The deviation is smaller than $4 \%$ with respect to the calibration with pure DEF. Therefore, the deviation due to fluid dependence is reduced by more than a factor of 10 compared to the state-of-the-art thermal flow sensor without fluid compensation.

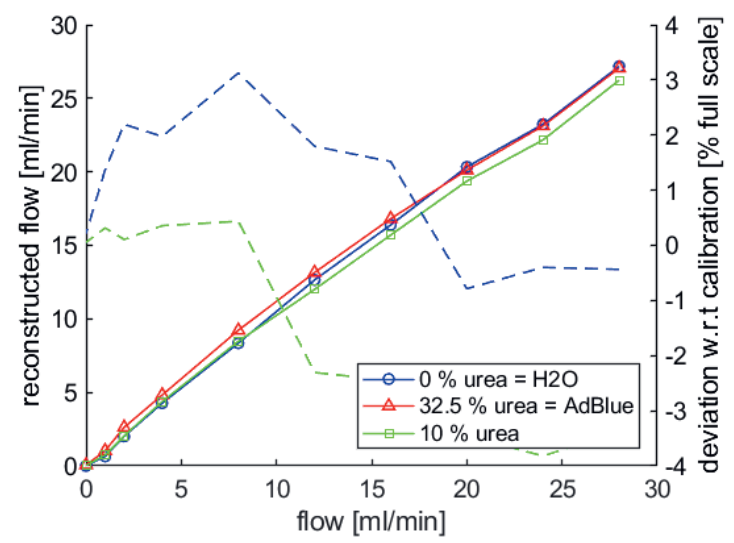

Figure 4: Linearized signal regarding flow, where the fluid dependence is reduced. Solid lines correspond to the linearized signal, dashed lines correspond to the deviation being smaller than $4 \%$ full scale with respect to the calibration curve of pure DEF.

\section{References}

[1] N. Kumawat, et al., Quality monitoring of diesel exhaust fluid in vehicles using diffractive interference sensors, SENSORS IEEE, 1676-1678 (2014); doi: 10.1109/ICSENS.2014.6985343

[2] P. Gurusamy, et al. Measurement of diesel exhaust fluid concentration in urea-SCR after-treatment system. Int. J. Precis. Eng. Manuf. 18, 1085-1092 (2017); doi: 10.1007/s12541-0170127-zFdgsf

[3] R.E. Bernhardsgrütter, et al., Inline Quality Monitoring of Diesel Exhaust Fluid (AdBlue) by Using the 3w-Method, SMSI 2020, 79-80 (2020); doi: 10.5162/SMSI2020/A5.2

[4] S. Sosna, et al. A Temperature Compensation Circuit for Thermal Flow Sensors Operated in Constant-Temperature Mode, IEEE Transaction on Instrumentation and Measurement 59, 17151721 (2010); doi: 10.1109/TIM.2009.2025988

[5] D.G. Cahill, et al. Thermal conductivity of $\alpha-S I: H$ thin films, Phys. Rev. B 50, 6077-6081 (1994); doi: 10.1103/PhysRevB.50.6077 